\title{
SPACE LIKE STRONG UNIQUE CONTINUATION FOR SUBLINEAR PARABOLIC EQUATIONS
}

\author{
AGNID BANERJEE AND RAMESH MANNA
}

\section{Contents}

1. Introduction 1

2. Notations and Preliminaries 5

3. Proof of the main results Theorem 1.2 and Theorem $1.1 \quad 8$

Proof of Theorem $1.2 \quad 8$

Proof of Theorem $1.1 \quad 15$

$\begin{array}{ll}\text { References } & 22\end{array}$

\begin{abstract}
In this paper, we establish space like strong unique continuation property (sucp) for uniformly parabolic sublinear equations under appropriate structural assumptions. Our main result Theorem 1.1 constitutes the parabolic counterpart of the strong unique continuation result recently established in $[\mathrm{Ru}]$ for analogous elliptic sublinear equations. Similar to that in [Ru], this is accomplished via a new $L^{2}-L^{2}$ type Carleman estimate for a class of sublinear parabolic operators.
\end{abstract}

\section{INTRODUCTION}

The primary objective of this paper is to study space like strong unique continuation for backward sublinear second order parabolic operators as in (1.8) below with structural assumptions on the sublinearity as in (1.9). To begin with, we note that an operator $L$ (local or non-local) is said to possess the strong unique continuation property if any non-trivial solution $u$ to

$$
L u=0
$$

in a (connected) domain $\Omega \subset \mathbb{R}^{n}$ cannot vanish to infinite order at any point in $\Omega$. An operator $L$ instead is said to have the weak unique continuation property (wucp) if a non-trivial solution to $L u=0$ cannot vanish in an open subset. Likewise, space-like strong unique continuation property for a parabolic operator $L$ asserts that if a solution $u$ to

$$
L u=0
$$

vanishes to infinite order at some point $\left(x_{0}, t_{0}\right)$, then $u\left(\cdot, t_{0}\right) \equiv 0$. The unique continuation property for second order elliptic and parabolic equations has a long history and by now has several important ramifications.

A prototypical example of an operator $L$ which satisfies the strong unique continuation property is the Laplacian $\Delta$ in which case sucp is a consequence of the real analyticity of solutions to

$$
\Delta u=0 .
$$

Second author supported by SERB National Postdoctoral fellowship, PDF/2017/0027. 
This property is however true for more general elliptic equations of the type

$$
\operatorname{div}(A(x) \nabla u)+b \cdot \nabla u+V u=0
$$

where the principal part $A$ can be allowed to be Lipschitz and where $b, V$ have appropriate integrability properties. Based on a visionary work due to Carleman in 1939 ( see [Car]) who established strong unique continuation for

$$
-\Delta+V, V \in L^{\infty}
$$

in $\mathbb{R}^{2}$, Carleman estimates were developed systematically in the seminal work of [AKS] in 1962 where sucp was established for Lipchitz $A$ and bounded $b, V$. We note that Lipschitz regularity assumption on $A$ is optimal in view of a deep counterexample due to Plis in [Pl]. Some of the other important works in this direction are due to Chanillo-Sawyer ([CS]), Kenig-Ruiz-Sogge $([\mathrm{KRS}])$ and Jerison-Kenig $([\mathrm{JK}])$. Each of these works deal with scaling critical potentials in different function spaces. For instance in [JK], sucp is established for

$$
-\Delta+V
$$

where $V \in L^{n / 2}$. The result of Jerison and Kenig was subsequently extended by Koch and Tataru in [KT0] to equations of the type (1.1) with borderline Lipschitz principal part.

An alternate approach which is instead based on the almost monotonicity of a generalized frequency function introduced by Almgren in [Al] came up in the works of Garofalo and Lin in 1986( [GL1], [GL2]). Using this approach, they were able to obtain new quantitative information on the zero set of solutions to divergence form elliptic equations and in particular, their results encompassed that of $[\mathrm{AKS}]$. Also in recent times, their approach found application in the optimal regularity of solutions for a class of free boundary problems known as Signorini problems (see for instance $[\mathrm{ACS}],[\mathrm{CSS}])$.

The study of weak unique continuation for parabolic equations began with the early work of Mizohata in [Mi] and Yamabe in [Y] followed by the work of Sogge in [So] where certain classes of unbounded potentials were treated using appropriate $L^{p}$ Carleman estimates. The study of strong backward uniqueness for parabolic equations for time independent coefficients began with the work of Lin in [L]. Subsequently Poon in [Po] established strong backward uniqueness for global solutions under Tychonoff type exponential growth assumption on the solution by adapting to the parabolic setting the frequency function approach of Garofalo and Lin. This continued with the work of Chen in [Ch] where instead Carleman estimates were employed. We note that backward uniqueness is in general not true without such global assumptions on the solution. This follows from a counterexample due to Frank Jones in [F] were it is shown that there exists a non-trivial unbounded caloric function that is supported in a time strip of the type $\mathbb{R}^{n} \times\left(t_{1}, t_{2}\right)$. Moreover, the counterexample of Frank Jones also shows that in general one cannot expect space-time strong unique continuation property for parabolic equations. Therefore in this scenario, the question of space like strong unique continuation is more relevant for local solutions.

This was taken up by Escauriaza and Fernandez in $[\mathrm{EF}]$ where they established space like strong unique continuation property for backward parabolic equations of the type

$$
\operatorname{div}(A(x, t) \nabla u)+\partial_{t} u+b(x, t) \cdot \nabla u+V u=0
$$

where $b, V$ are bounded and the principal part $A$ has regularity assumptions similar to that in (1.11) below. We also refer to the subsequent work of Escauriaza-Fernandez-Vessella in [EFV] 
where certain quantitative results were obtained. The approach in $[\mathrm{EF}]$ and $[\mathrm{EFV}]$ are based on a $L^{2}-L^{2}$ type Carleman estimate of the type

$$
\left\|t^{-\alpha-1 / 2} e^{-\frac{|x|^{2}}{8 t}} u\right\|_{L^{2}} \lesssim\left\|t^{-\alpha} e^{-\frac{|x|^{2}}{8 t}}\left(\partial_{t} u+\operatorname{div}(A \nabla u)\right)\right\|_{L^{2}}
$$

which is obtained using a fairly nontrivial parabolic Rellich type identity as stated in Lemma 2.1 below coupled with a clever integration by parts argument. The Carleman estimate in [EF] is also partly inspired by the previous work of Poon in [Po]. In fact the work of Poon contributed in clarifying the correct form of Carleman estimates that can be expected in the parabolic situation. The space like sucp in $[\mathrm{EF}]$ was later on extended in $[\mathrm{KT}]$ to parabolic equations with principal part $A(x, t)$ having lower regularity in the time variable $t$ and where the lower order terms $b$ and $V$ are allowed to belong to some scaling critical function spaces similar to that in the elliptic case as in [JK] and [KT0]. We note that unlike that in $[\mathrm{EF}]$, the proof in $[\mathrm{KT}]$ is instead based on deep $L^{p}$ spectral projection bounds for the Hermite operator. Such bounds were independently obtained by Thangavelu in [T] and Kharazdhov in $[\mathrm{K}]$ and they were also essential in the proof of $L^{p}$ Carleman estimates for the heat operator in the previous works of Escauriaza in $[\mathrm{E}]$ and Escauriaza and Vega in $[\mathrm{EV}]$ using which the authors showed backward uniqueness for (1.2) when $A=\mathbb{I}$ and $V \in L^{1} L^{\infty}+L^{\infty} L^{n / 2}$.

Now regarding sublinear equations, we note that motivated by the study of nonlinear eigenvalue problems, the analysis of corresponding nodal domains as in $[\mathrm{PW}]$ and also because of certain connection of such equations to porous media type equations (see for instance [Vaz]), the study of unique continuation for sublinear elliptic equations was taken up in recent times by Soave and Weth in [SW] where they established wucp for equations of the type

$$
\operatorname{div}(A(x) \nabla u)+f(x, u)+V u=0
$$

where the sublinear term $f$ satisfies the structural assumptions similar to that in (1.9) below. Such equations are modeled on

$$
-\Delta u=|u|^{p-2} u \text {. }
$$

Note that the study of strong unique continuation for (1.5) cannot be reduced to that for

$$
-\Delta+V
$$

because in this case, $V=|u|^{p-2}$ need not be in $L^{n / 2}$ near the zero set of $u$ as $p \in(1,2)$. In fact such sublinear equations have their intrinsic difficulties and this is also partly visible from the fact that the sign assumption on the sublinearity $f$ in (1.9) is quite crucial because otherwise unique continuation fails. This later fact follows from a counterexample in [SW] where it is shown that unique continuation is not true for

$$
\Delta u=|u|^{p-2} u, p \in(1,2) .
$$

In [SW], the authors adapted the frequency function approach of Garofalo and Lin and also that of Garofalo and Smit Vega Garcia as in [GG]. The question of strong unique continuation for such sublinear equations was then later addressed by Ruland in [Ru] via new Carleman estimates for the corresponding sublinear elliptic operators. See also the recent interesting work of Soave and Terracini in $[\mathrm{ST}]$ where the authors study the following two phase membrane problem

$$
-\Delta u=\lambda_{+}\left(u^{+}\right)^{q-1}-\lambda_{-}\left(u^{-}\right)^{q-1}, \text { where } \lambda_{+}, \lambda_{-}>0, q \in[1,2)
$$

and establish a strong unique continuation property as well as a regularity result for the nodal domains of solutions to such equations. The key object in their analysis is a monotonicity formula for a 2-parameter family of Weiss type functionals introduced by Weiss in [We]. The 
reader should however note that although (1.7) is a more general equation than (1.6), but it doesn't encompass the class of equations as in (1.4). Therefore the unique continuation results in $[\mathrm{Ru}]$ and $[\mathrm{SW}]$ are not covered by the results in $[\mathrm{ST}]$ and also vice-versa.

We would also like to refer to a recent work by two of us with Garofalo as in [BGR] where the result of Ruland has been extended to sublinear equations associated to degenerate elliptic Baouendi-Grushin operators $\mathbb{B}_{\gamma}$ defined by

$$
\mathbb{B}_{\gamma}=\Delta_{z}+|z|^{2 \gamma} \Delta_{t},(z, t) \in \mathbb{R}^{m} \times R^{n} .
$$

The method in [BGR] also slightly simplifies the proof of Ruland when the principal part is $\Delta$ and moreover our proof of the sublinear Carleman estimate as stated in (1.14) below is also inspired in parts by the ideas in [BGR]. We also note that the recent work [ST] addresses the related nodal domain estimates for solutions to such sublinear equations.

Therefore given the recent developments in the sublinear unique continuation theory in the elliptic case as in $[\mathrm{SW}]$ and $[\mathrm{Ru}]$, in this paper, we study analogous strong unique continuation for backward parabolic sublinear equations of the type

$$
\sum_{i, j=1}^{n} \partial_{i}\left(a_{i j}(x, t) \partial_{j} u\right)+\partial_{t} u+f(X, u)+V u=0
$$

where $V \in L^{\infty}$ and $f$ and its primitive $F$ satisfies the following structural assumptions similar to that in $[\mathrm{Ru}]$ and $[\mathrm{SW}]$ for some $\kappa, K>0$ :

$$
\left\{\begin{array}{l}
f((x, t), 0)=0 \\
F((x, t), s)=\int_{0}^{s} f(X, s) d s \\
0<s f((x, t), s) \leq q F((x, t), s), \text { for some } q \in(1,2) \text { and } s \in(-1,1) \backslash\{0\} \\
\left|\nabla_{(x, t)} f\right| \leq K|f|,\left|\nabla_{(x, t)} F\right| \leq K F \\
f((x, t), s) \leq \kappa s^{p-1} \text { for some } p \in(1,2) ;
\end{array}\right.
$$

We note that the first and the last condition in (1.9) implies that for some constant $c_{0}, c_{1}$, we have that

$$
c_{1} s^{p} \geq F(\cdot, s) \geq c_{0} s^{q}, \text { for } s \in(-1,1) .
$$

A prototypical $f$ satisfying (1.9) is given by

$$
f((x, t), u)=\sum_{i=1}^{l} c_{i}(x, t)|u|^{q_{i}-2} u,
$$

where for each $i, q_{i} \in(1,2), 0<k_{0}<c_{i}<k_{1}$ and $\left|\nabla c_{i}\right|<K$ for some $k_{0}, k_{1}$ and $K$. In this case, we can take $q=\max \left\{q_{i}\right\}$ and $p=\min \left\{q_{i}\right\}$. On the principal part $A=\left(a_{i j}\right)$, similar to [EF], we assume that there exists $C_{0}, \lambda>0$ and $0<\beta \leq 1$ such that for all $x$ and $y$ in $\mathbb{R}^{n}$ and $0 \leq t, s<\infty$, we have

$$
\left\{\begin{array}{l}
\lambda|\xi|^{2} \leq \sum a_{i j}((x, t), u) \xi_{i} \xi_{j} \leq \lambda^{-1}|\xi|^{2}, \text { for some } \lambda>0 \\
|A(x, t)-A(y, s)| \leq C_{0}\left(|x-y|^{2}+|t-s|\right)^{\beta / 2} \text { and also }\left|\nabla_{x} a_{i j}(x, t)\right| \leq C_{0}|x|^{\beta-1},\left|\partial_{t} a_{i j}(x, t)\right| \leq C_{0} t^{\beta / 2-1} .
\end{array}\right.
$$

A typical situation when (1.11) is satisfied is when the principal part $A$ is uniformly elliptic and Lipschitz continuous in both $x$ and $t$. Our main result which is the parabolic counterpart of the strong unique continuation result in $[\mathrm{Ru}]$ can now be stated as follows. 
Theorem 1.1. Let $u \in L^{\infty}\left(B_{2} \times[0,2)\right)$ be a solution to (1.8) in $B_{2} \times[0,2)$ where $V$ satisfies

$$
\|V\|_{L^{\infty}\left(B_{2} \times[0,2)\right)} \leq M
$$

and the coefficient matrix $\left(a_{i j}\right)$ satisfies the assumptions in (1.11).

Now if $u$ vanishes to infinite order in space in the sense of Definition 2.5 below, then we have that $u(x, 0)=0$ for all $x \in B_{2}$.

Similar to [Ru], our proof of Theorem 1.1 is based on the following new Carleman estimate for sublinear parabolic operators which in turn is based on a somewhat delicate adaptation of the techniques in $[\mathrm{EF}]$ to our sublinear situation. As the reader will see, the proof of the following estimate is made possible by combination of several non-trivial geometric facts which thanks to the specific structure of the sublinearity, beautifully combine. Moreover, unlike the elliptic case, the proof of Theorem 1.1 following the Carleman estimate is somewhat more involved because the ensuing inequalities are in the Gaussian space.

Theorem 1.2. For a given $\alpha \geq 1$, with $\gamma=\frac{\alpha}{\delta^{2}}$, let $u \in C_{0}^{\infty}\left(B_{2} \times\left(0, \frac{1}{2 \gamma}\right)\right)$ be a solution to

$$
\operatorname{div}(A \nabla u)+\partial_{t} u+f((x, t), u)=g, 1<q<2,
$$

where the coefficient matrix $A=\left(a_{i j}\right)$ satisfies (1.11). Define $\sigma$ as in Lemma 2.3 below corresponding to $\theta$ as in (2.2) and $\gamma$ as above. Also let $G$ be as in Lemma 2.4. Then there are numbers $\delta_{0}, N_{0}$ and $\tilde{C}$ depending on $\lambda, C_{0}, \beta$ in (1.11) as well as the parameters in (1.9) such that for $\alpha \geq \tilde{C}$ and $\delta \leq \delta_{0}$, the following inequality holds,

$$
\begin{aligned}
& \alpha \int_{\mathbb{R}_{+}^{n+1}} \sigma^{-\alpha} \frac{\theta(\gamma t)}{t}|u|^{2} G d X+\int_{\mathbb{R}_{+}^{n+1}} \sigma^{1-\alpha} \frac{\theta(\gamma t)}{t}|\nabla u|^{2} G d X \\
& +O(\alpha) \int_{\mathbb{R}_{+}^{n+1}} \sigma^{-\alpha} F(X, u) G d X \\
& \leq N_{0} \int_{\mathbb{R}_{+}^{n+1}} \sigma^{1-\alpha}|g|^{2} G d X+e^{N_{0} \alpha} \gamma^{\alpha+N_{0}} \int_{\mathbb{R}_{+}^{n+1}}\left(u^{2}+t|\nabla u|^{2}+F(X, u)\right) d X .
\end{aligned}
$$

In closing, we would like to mention that it remains to be seen whether one can also establish a backward uniqueness result for sublinear equations of the type (1.8) under global growth assumptions on the solution similar to that in [Po], [Ch], [ESS] and [WZ]. It also seems to be a challenging open problem as to whether the regularity assumptions on the principal part $A$ in Theorem 1.1 can be further relaxed as in $[\mathrm{KT}]$. We would like to address such questions in a future study.

The paper is organized as follows. In Section 2, we introduce some basic notations and gather some known results that are relevant to our work. In Section 3, we finally prove our main results.

\section{Notations And Preliminaries}

In this section we introduce some basic notations and also collect some background results from [EF] which will be used throughout our work. Given $r>0$ we denote by $B_{r}\left(x_{0}\right)$ the Euclidean ball centered at $x_{0} \in \mathbb{R}^{n}$ and when $x_{0}=0$, we denote it simply by $B_{r}$. From now on, a generic point $(x, t)$ in $\mathbb{R}^{n} \times[0, \infty)$ denoted by $X$. Also, unless and otherwise specified, $\nabla U$, div $U$ will refer to $\nabla_{x} U$, $\operatorname{div}_{x} U$ respectively. The region $\mathbb{R}^{n} \times(0, \infty)$ in space-time will be denoted by $\mathbb{R}_{+}^{n+1}$. The notation $A \lesssim B$ would be mean $A \leq C B$ for some universal $C$. Also, for a given a function $\sigma(t), \dot{\sigma}(t)$ would refer to its derivative. 
We now state the relevant results from $[\mathrm{EF}]$. The first lemma is a parabolic Rellich type identity which corresponds to Lemma 1 in $[\mathrm{EF}]$ and similar to that in [EF] and [EFV], constitutes the key ingredient in the proof of our sublinear Carleman estimate.

Lemma 2.1. Let $\sigma=\sigma(t)$ be a non-decreasing function satisfying $\sigma(0)=0, \alpha \in \mathbb{R}$, and $H$ and $G$ denote two functions in $\mathbb{R}_{+}^{n+1}, G$ non-negative. Then, the following identity holds for all $u \in C_{0}^{\infty}\left(\mathbb{R}_{+}^{n+1}\right)$,

$$
\begin{aligned}
& 2 \int_{\mathbb{R}_{+}^{n+1}} \frac{\sigma^{1-\alpha}}{\dot{\sigma}}\left(\partial_{t} u-<A \nabla \log G, \nabla u>+\frac{1}{2} H u-\frac{\alpha \dot{\sigma}}{2 \sigma} u\right)^{2} G d X \\
& +\int_{\mathbb{R}_{+}^{n+1}} \frac{\sigma^{1-\alpha}}{\dot{\sigma}} \partial_{t}\left[\log \frac{\sigma}{\dot{\sigma} t}\right]<A \nabla u, \nabla u>G d X \\
& =2 \int_{\mathbb{R}_{+}^{n+1}} \frac{\sigma^{1-\alpha}}{\dot{\sigma}}\left(\operatorname{div}(A \nabla u)+\partial_{t} u\right)\left(\partial_{t} u-<A \nabla \log G, \nabla u>+\frac{1}{2} H u-\frac{\alpha \dot{\sigma}}{2 \sigma} u\right) G d X \\
& +\int_{\mathbb{R}_{+}^{n+1}} \frac{\sigma^{1-\alpha}}{\dot{\sigma}} u<A \nabla u, \nabla H>G d X-\frac{1}{2} \int_{\mathbb{R}_{+}^{n+1}} \frac{\sigma^{1-\alpha}}{\dot{\sigma}} u^{2} M d X \\
& +\frac{\alpha}{2} \int_{\mathbb{R}_{+}^{n+1}} \sigma^{-\alpha} u^{2}\left(\partial_{t} G-\operatorname{div}(A \nabla G)-H G\right) d X \\
& -\int_{\mathbb{R}_{+}^{n+1}} \frac{\sigma^{1-\alpha}}{\dot{\sigma}}<A \nabla u, \nabla u>\left(\partial_{t} G-\operatorname{div}(A \nabla G)-H G\right) d X-2 \int_{\mathbb{R}_{+}^{n+1}} \frac{\sigma^{1-\alpha}}{\dot{\sigma}} D_{G} \nabla u \cdot \nabla u d X
\end{aligned}
$$

where

$$
M=\partial_{t}\left[\log \frac{\sigma}{\dot{\sigma} t}\right] H G+\partial_{t} H G+H\left(\partial_{t} G-\operatorname{div}(A \nabla G)-H G\right)-<A \nabla G, \nabla H>;
$$

$D_{G}$ is the $n \times n$ symmetric matrix defined as

$D_{G}^{i j}=\frac{a_{i j}}{2 t} G+a_{i l} \partial_{k l} G a_{k j}-\frac{a_{i k} \partial_{k} G a_{j l} \partial_{l} G}{G}+\frac{1}{2} \partial_{k} a_{i l} \partial_{l} G a_{k j}+\frac{1}{2} \partial_{k} a_{j l} \partial_{l} G a_{k i}-\frac{1}{2} a_{k l} \partial_{l} G \partial_{k} a_{i j}+\frac{1}{2} \partial_{t} a_{i j} G$.

We also need the following identity ( see Lemma 2 in $[\mathrm{EF}]$ ).

Lemma 2.2. Assume that $\sigma$ and $G$ are as in Lemma 2.1. Then, the following identity holds for $u \in C_{0}^{\infty}\left(\mathbb{R}_{+}^{n+1}\right)$ and $\alpha \in \mathbb{R}$,

$$
\begin{aligned}
(\alpha-1) \int_{\mathbb{R}_{+}^{n+1}} \sigma^{-\alpha} \partial_{t}\left[\log \frac{\sigma}{\dot{\sigma} t}\right] u^{2} G d X & =2 \int_{\mathbb{R}_{+}^{n+1}} \frac{\sigma^{1-\alpha}}{\dot{\sigma}} \partial_{t}\left[\log \frac{\sigma}{\dot{\sigma} t}\right]\left[u\left(\operatorname{div}(A \nabla u)+\partial_{t} u\right)+|\nabla u|^{2}\right] G d X \\
& +2 \int_{\mathbb{R}_{+}^{n+1}} \frac{\sigma^{1-\alpha}}{\dot{\sigma}} \partial_{t}\left[\log \frac{\sigma}{\dot{\sigma} t}\right] u^{2}\left(\partial_{t} G-\operatorname{div}(A \nabla G)\right) d X \\
& -2 \int_{\mathbb{R}_{+}^{n+1}} \sigma^{1-\alpha} \partial_{t}\left(\frac{1}{\dot{\sigma}} \partial_{t}\left[\log \frac{\sigma}{\dot{\sigma} t}\right]\right) u^{2} G d X .
\end{aligned}
$$

As in $[\mathrm{EF}], \sigma$ as in (1.14) is chosen to be a solution to an appropriate ordinary differential equation which is dictated by the identity above. To this end, we have the following Lemma which is Lemma 4 in $[\mathrm{EF}]$. 
Lemma 2.3. Assume that $\theta:(0,1) \rightarrow \mathbb{R}_{+}$satisfies

$$
0 \leq \theta \leq N,|t \dot{\theta}(t)| \leq N \theta(t) \text { and } \int_{0}^{1}\left(1+\log \frac{1}{t}\right) \frac{\theta(t)}{t} d t \leq N
$$

for some constant $N$. Then the solution to the ordinary differential equation

$$
\frac{d}{d t} \log \left(\frac{\sigma}{t \dot{\sigma}}\right)=\frac{\theta(\gamma t)}{t}, \sigma(0)=0, \dot{\sigma}(0)=1,
$$

where $\gamma>0$, has the following properties when $0 \leq \gamma t \leq 1$ :

(1) $t e^{-N} \leq \sigma(t) \leq t$

(2) $e^{-N} \leq \dot{\sigma}(t) \leq 1$,

(3) $\left|\partial_{t}\left[\sigma \log \frac{\sigma}{\dot{\sigma} t}\right]\right|+\left|\partial_{t}\left[\sigma \log \frac{\sigma}{\dot{\sigma}}\right]\right| \leq 3 N$,

(4) $\left|\sigma \partial_{t}\left(\frac{1}{\dot{\sigma}} \partial_{t}\left[\log \frac{\sigma}{\dot{\sigma} t}\right]\right)\right| \leq 3 N e^{N} \frac{\theta(\gamma t)}{t}$.

Now corresponding to $\beta$ as in (1.11), the function $\theta$ is chosen as follows

$$
\theta(t)=t^{\beta / 2}\left(\log \frac{1}{t}\right)^{1+\beta / 2}
$$

It is easily seen that $\theta$ satisfies the conditions in Lemma 2.3. From now, let $0<\delta<1$ denote a small number to be chosen later, and $\alpha$ and $\beta$ be two numbers satisfying $\alpha \geq 1$ and $0<\beta \leq 1$. We also need the following weighted inequalities in the Gaussian space ( see Lemma 5 in $[\mathrm{EF}]$ ).

Lemma 2.4. Let $G(X)=t^{-n / 2} e^{-|x|^{2} / 4 t}$ and $\sigma$ denote the function defined in Lemma 2.3 corresponding to $\gamma=\frac{\alpha}{\delta^{2}}$ and $\theta$ as in $(2.2)$.

Then, there is a constant $N$ depending on $\beta$ and $n$ such that the following inequalities hold for all functions $u \in C_{0}^{\infty}\left(\mathbb{R}^{n} \times[0,1 / 2 \gamma)\right)$,

$$
\begin{aligned}
& \int_{\mathbb{R}_{+}^{n+1}} \sigma^{-\alpha} u^{2}\left(\frac{|x|^{\beta}}{t}+\frac{|x|^{2+\beta}}{\alpha t^{2}}+t^{\beta / 2-1}\right) G d X \\
& \leq N e^{N \alpha} \gamma^{\alpha+N} \int_{\mathbb{R}_{+}^{n+1}} u^{2} d X+N \delta^{\beta} \int_{\mathbb{R}_{+}^{n+1}} \sigma^{-\alpha} \frac{\theta(\gamma t)}{t} u^{2} G d X ; \\
& \int_{\mathbb{R}_{+}^{n+1}} \sigma^{1-\alpha}|\nabla u|^{2}\left(\frac{|x|^{\beta}}{t}+\frac{|x|^{2+\beta}}{\alpha t^{2}}+\frac{|x|^{1+\beta}}{t \delta}+t^{\beta / 2-1}\right) G d X \\
& \leq N e^{N \alpha} \gamma^{\alpha+N} \int_{\mathbb{R}_{+}^{n+1}} t|\nabla u|^{2} d X+N \delta^{\beta} \int_{\mathbb{R}_{+}^{n+1}} \sigma^{1-\alpha} \frac{\theta(\gamma t)}{t}|\nabla u|^{2} G d X .
\end{aligned}
$$

In closing, we define the relevant notion of vanishing to infinite order in space.

Definition 2.5. We say that a function u defined in a region $\Omega$ in space time vanishes to infinite order in space at $\left(x_{0}, t_{0}\right) \in \Omega$ if given $k>0$, there exists $C_{k}>0$ such

$$
\left|u\left(x, t_{0}\right)\right| \leq C_{k}\left|x-x_{0}\right|^{k}
$$

for all $\left(x, t_{0}\right) \in \Omega$. 


\section{Proof of the main Results Theorem 1.2 And Theorem 1.1}

\section{Proof of Theorem 1.2.}

Proof. By rotation of coordinates, without loss of generality we may assume that $A(0,0)=\mathbb{I}$. Then as in $[\mathrm{EF}]$, we let $r(x)=|x|$ and

$$
H=\frac{r^{2}(1-<A \nabla r, \nabla r>)}{4 t^{2}} .
$$

By a standard calculation we have

$$
\partial_{t} G-\operatorname{div}(A \nabla G)=\left(\frac{r^{2}(1-<A \nabla r, \nabla r>)}{4 t^{2}}+\frac{(<A \nabla r, \nabla r>-1)}{2 t}+\frac{r \operatorname{div}(A \nabla r)-(n-1)}{2 t}\right) G
$$

and

$$
|H| \lesssim \min \left(\frac{\left(|x|^{2}+t\right)^{1+\beta / 2}}{t^{2}}, \frac{|x|^{2}}{t^{2}}\right),\left|\partial_{t} G-\operatorname{div}(A \nabla G)-H G\right| \lesssim \frac{\left(|x|^{2}+t\right)^{\beta / 2}}{t} G
$$

Note that (3.1) in particular implies that

$$
\left|\partial_{t} G-\operatorname{div}(A \nabla G)\right| \lesssim\left(\frac{\left(|x|^{2}+t\right)^{1+\beta / 2}}{t^{2}}+\frac{\left(|x|^{2}+t\right)^{\beta / 2}}{t}\right) G
$$

Now as in the statement of Theorem 1.2, for a given $\alpha$, we have

$$
\gamma=\alpha / \delta^{2} .
$$

Let $\theta, \sigma$ be also as in Theorem 1.2. Then by using the identity in Lemma 2.2 and the equation (1.13) satisfied by $u$, the estimates in (3.2) and the bounds for $\sigma, \dot{\sigma}$ in Lemma 2.3, we get the following estimate

$$
\begin{aligned}
& (\alpha-1) \int_{\mathbb{R}_{+}^{n+1}} \sigma^{-\alpha} \frac{\theta(\gamma t)}{t} u^{2} G d X \\
& \leq 2 \int_{\mathbb{R}_{+}^{n+1}} \frac{\sigma^{1-\alpha}}{\dot{\sigma}} \frac{\theta(\gamma t)}{t} u(-f(X, u)+g) G d X \\
& +C\left[\int_{\mathbb{R}_{+}^{n+1}} \frac{\sigma^{1-\alpha}}{\dot{\sigma}} \frac{\theta(\gamma t)}{t}\left(|\nabla u|^{2}+u^{2}\left(\frac{\left(|x|^{2}+t\right)^{1+\beta / 2}}{t^{2}}+\frac{\left(|x|^{2}+t\right)^{\beta / 2}}{t}\right)\right) G d X\right. \\
& \left.+\int_{\mathbb{R}_{+}^{n+1}} \sigma^{-\alpha} \frac{\theta(\gamma t)}{t} u^{2} G d X\right] .
\end{aligned}
$$

Note that in (3.3) above, we also used the differential equation satisfied by $\sigma$ as in Lemma 2.3. Now since $\frac{\theta(\gamma t)}{t} \lesssim \sigma^{-1}$, therefore by applying the weighted inequality in Lemma 2.4 to the term

$$
\int_{\mathbb{R}_{+}^{n+1}} \frac{\sigma^{1-\alpha}}{\dot{\sigma}} \frac{\theta(\gamma t)}{t} u^{2}\left(\frac{\left(|x|^{2}+t\right)^{1+\beta / 2}}{t^{2}}+\frac{\left(|x|^{2}+t\right)^{\beta / 2}}{t}\right) G d X
$$


we deduce that the following holds

$$
\begin{aligned}
& (\alpha-1) \int_{\mathbb{R}_{+}^{n+1}} \sigma^{-\alpha} \frac{\theta(\gamma t)}{t} u^{2} G d X \\
& \leq C\left[\int_{\mathbb{R}_{+}^{n+1}} \sigma^{1-\alpha} \frac{\theta(\gamma t)}{t}|\nabla u|^{2} G d X+e^{N \alpha} \gamma^{\alpha+N} \int_{\mathbb{R}_{+}^{n+1}} u^{2} d X+\left(\alpha \delta^{\beta}+1\right) \int_{\mathbb{R}_{+}^{n+1}} \sigma^{-\alpha} \frac{\theta(\gamma t)}{t} u^{2} G d X\right] \\
& +2 \int_{\mathbb{R}_{+}^{n+1}} \frac{\sigma^{1-\alpha}}{\dot{\sigma}} \frac{\theta(\gamma t)}{t} u(-f(X, u)+g) G d X
\end{aligned}
$$

for some universal $C, N$ depending also on the bounds in (1.11). Now observe that if $\delta$ is small enough and $\alpha$ is taken large enough, then the following integral in (3.4)

$$
C\left(\alpha \delta^{\beta}+1\right) \int_{\mathbb{R}_{+}^{n+1}} \sigma^{-\alpha} \frac{\theta(\gamma t)}{t} u^{2} G d X
$$

can be absorbed in the left hand side. Moreover since

$$
u f(X, u) \geq 0
$$

the following integral on the right hand side of (3.4)

$$
2 \int_{\mathbb{R}_{+}^{n+1}} \frac{\sigma^{1-\alpha}}{\dot{\sigma}} \frac{\theta(\gamma t)}{t} u(-f(X, u)) G
$$

is non-positive and hence the inequality in (3.4) remains valid without this term. Then by applying Cauchy Schwartz inequality to

$$
2 \int_{\mathbb{R}_{+}^{n+1}} \frac{\sigma^{1-\alpha}}{\dot{\sigma}} \frac{\theta(\gamma t)}{t} u g G d X
$$

we deduce from (3.4) that the following estimate holds,

$$
\begin{aligned}
& \alpha \int_{\mathbb{R}_{+}^{n+1}} \sigma^{-\alpha} \frac{\theta(\gamma t)}{t} u^{2} G d X \\
& \leq C_{1}\left[\int_{\mathbb{R}_{+}^{n+1}} \sigma^{1-\alpha} \frac{\theta(\gamma t)}{t}|\nabla u|^{2} G d X+\int_{\mathbb{R}_{+}^{n+1}} \sigma^{1-\alpha}|g|^{2} G d X\right. \\
& \left.+e^{N \alpha} \gamma^{\alpha+N} \int_{\mathbb{R}_{+}^{n+1}} u^{2} d X\right]
\end{aligned}
$$

for some $C_{1}$ universal. Now in order to incorporate the gradient term on the left hand side in the Carleman estimate, we make use of the identity in Lemma 2.1. For that, we first note that using

$$
\frac{\delta_{i j}}{2 t} G+\partial_{i j} G-\frac{\partial_{i} G \partial_{j} G}{G}=0
$$

and the bounds on the derivatives of $A$ as in (1.11) that the following estimate holds,

$$
\left|D_{G} \nabla u \cdot \nabla u\right| \lesssim \frac{\left(|x|^{2}+t\right)^{\beta / 2}}{t}|\nabla u|^{2} G
$$


This corresponds to the estimate (3.3) in $[\mathrm{EF}]$. Next from Lemma 2.3, the bounds on the coefficients as in (1.11) and (3.1), we have

$$
|\sigma \nabla H| \lesssim \frac{\left(|x|^{2}+t\right)^{1+\beta / 2}}{t} \text { and }|\sigma M| \lesssim \frac{\left(|x|^{2}+t\right)^{1+\beta / 2}}{t^{2}} G
$$

Then by applying the identity as in Lemma 2.1 and by using the equation (1.13) satisfied by $u$ we obtain

$$
\begin{aligned}
& 2 \int_{\mathbb{R}_{+}^{n+1}} \frac{\sigma^{1-\alpha}}{\dot{\sigma}}\left(\partial_{t} u-<A \nabla \log G, \nabla u>+\frac{1}{2} H u-\frac{\alpha \dot{\sigma}}{2 \sigma} u\right)^{2} G d X \\
& +\int_{\mathbb{R}_{+}^{n+1}} \frac{\sigma^{1-\alpha}}{\dot{\sigma}} \partial_{t}\left[\log \frac{\sigma}{\dot{\sigma} t}\right]<A \nabla u, \nabla u>G d X \\
& =2 \int_{\mathbb{R}_{+}^{n+1}} \frac{\sigma^{1-\alpha}}{\dot{\sigma}}(-f(X, u)+g)\left(\partial_{t} u-<A \nabla \log G, \nabla u>+\frac{1}{2} H u-\frac{\alpha \dot{\sigma}}{2 \sigma} u\right) G d X \\
& +\int_{\mathbb{R}_{+}^{n+1}} \frac{\sigma^{1-\alpha}}{\dot{\sigma}} u<A \nabla u, \nabla H>G d X-\frac{1}{2} \int_{\mathbb{R}_{+}^{n+1}} \frac{\sigma^{1-\alpha}}{\dot{\sigma}} u^{2} M d X \\
& +\frac{\alpha}{2} \int_{\mathbb{R}_{+}^{n+1}} \sigma^{-\alpha} u^{2}\left(\partial_{t} G-\operatorname{div}(A \nabla G)-H G\right) d X \\
& -\int_{\mathbb{R}_{+}^{n+1}} \frac{\sigma^{1-\alpha}}{\dot{\sigma}}<A \nabla u, \nabla u>\left(\partial_{t} G-\operatorname{div}(A \nabla G)-H G\right) d X-2 \int_{\mathbb{R}_{+}^{n+1}} \frac{\sigma^{1-\alpha}}{\dot{\sigma}} D_{G} \nabla u \cdot \nabla u d X .
\end{aligned}
$$

Now, using Cauchy-Schwarz inequality, the following integral on the right hand side

$$
2 \int_{\mathbb{R}_{+}^{n+1}} \frac{\sigma^{1-\alpha}}{\dot{\sigma}} g\left(\partial_{t} u-<A \nabla \log G, \nabla u>+\frac{1}{2} H u-\frac{\alpha \dot{\sigma}}{2 \sigma} u\right) G d X
$$

can be estimated as

$$
\begin{aligned}
& 2 \int_{\mathbb{R}_{+}^{n+1}} \frac{\sigma^{1-\alpha}}{\dot{\sigma}} g\left(\partial_{t} u-<A \nabla \log G, \nabla u>+\frac{1}{2} H u-\frac{\alpha \dot{\sigma}}{2 \sigma} u\right) G d X \\
& \leq \frac{1}{4} \int_{\mathbb{R}_{+}^{n+1}} \frac{\sigma^{1-\alpha}}{\dot{\sigma}}\left(\partial_{t} u-<A \nabla \log G, \nabla u>+\frac{1}{2} H u-\frac{\alpha \dot{\sigma}}{2 \sigma} u\right)^{2} G d X \\
& +8 \int_{\mathbb{R}_{+}^{n+1}} \frac{\sigma^{1-\alpha}}{\dot{\sigma}} g^{2} G d X
\end{aligned}
$$

and then the first integral in the right hand side of (3.9) can be absorbed into the first term in the left hand side of (3.8). Consequently it follows from (3.8) and (3.9) and by using the bounds 
(3.1), (3.6), (3.7) as well as the inequalities in Lemma 2.4 that the following estimate holds

$$
\begin{aligned}
& \int_{\mathbb{R}_{+}^{n+1}} \sigma^{1-\alpha} \frac{\theta(\gamma t)}{t}|\nabla u|^{2} G d X \\
& \leq \int_{\mathbb{R}_{+}^{n+1}} \frac{\sigma^{1-\alpha}}{\dot{\sigma}}(-f(X, u))\left(\partial_{t} u-<A \nabla \log G, \nabla u>+\frac{1}{2} H u-\frac{\alpha \dot{\sigma}}{2 \sigma} u\right) G d X \\
& +C\left[\int_{\mathbb{R}_{+}^{n+1}} \sigma^{1-\alpha}|g|^{2} G d X+\alpha \delta^{\beta} \int_{\mathbb{R}_{+}^{n+1}} \sigma^{-\alpha} \frac{\theta(\gamma t)}{t}|u|^{2} G d X\right. \\
& \delta^{\beta} \int_{\mathbb{R}_{+}^{n+1}} \sigma^{1-\alpha} \frac{\theta(\gamma t)}{t}|\nabla u|^{2} G d X+\int_{\mathbb{R}_{+}^{n+1}} \sigma^{-\alpha}|u||\nabla u| \frac{\left(|x|^{2}+t\right)^{(1+\beta) / 2}}{t} G d X \\
& \left.+e^{N \alpha} \gamma^{\alpha+N} \int_{\mathbb{R}_{+}^{n+1}}\left(u^{2}+t|\nabla u|^{2}\right) d X\right] .
\end{aligned}
$$

Finally the term

$$
\int_{\mathbb{R}_{+}^{n+1}} \sigma^{-\alpha}|u||\nabla u| \frac{\left(|x|^{2}+t\right)^{(1+\beta) / 2}}{t} G d X
$$

is handled using Cauchy-Schwarz inequality in the following way

$$
\begin{aligned}
\int_{\mathbb{R}_{+}^{n+1}} \sigma^{-\alpha}|u||\nabla u| \frac{\left(|x|^{2}+t\right)^{(1+\beta) / 2}}{t} G d X & \lesssim \int_{\mathbb{R}_{+}^{n+1}} \sigma^{-\alpha}|u|^{2} \frac{\left(|x|^{2}+t\right)^{1+\beta / 2}}{t^{2}} G d X \\
& +\int_{\mathbb{R}_{+}^{n+1}} \sigma^{1-\alpha}|\nabla u|^{2} \frac{\left(|x|^{2}+t\right)^{\beta / 2}}{t} G d X .
\end{aligned}
$$

Now the terms on the right hand side of (3.11) are again estimated using the inequalities in Lemma 2.4 and consequently we deduce from (3.10) that the following holds

$$
\begin{aligned}
& \int_{\mathbb{R}_{+}^{n+1}} \sigma^{1-\alpha} \frac{\theta(\gamma t)}{t}|\nabla u|^{2} G d X \\
& \leq \int_{\mathbb{R}_{+}^{n+1}} \frac{\sigma^{1-\alpha}}{\dot{\sigma}}(-f(X, u))\left(\partial_{t} u-<A \nabla \log G, \nabla u>+\frac{1}{2} H u-\frac{\alpha \dot{\sigma}}{2 \sigma} u\right) G d X \\
& +C\left[\int_{\mathbb{R}_{+}^{n+1}} \sigma^{1-\alpha}|g|^{2} G d X+\alpha \delta^{\beta} \int_{\mathbb{R}_{+}^{n+1}} \sigma^{-\alpha} \frac{\theta(\gamma t)}{t}|u|^{2} G d X\right. \\
& \left.\delta^{\beta} \int_{\mathbb{R}_{+}^{n+1}} \sigma^{1-\alpha} \frac{\theta(\gamma t)}{t}|\nabla u|^{2} G d X+e^{N \alpha} \gamma^{\alpha+N} \int_{\mathbb{R}_{+}^{n+1}}\left(u^{2}+t|\nabla u|^{2}\right) d X\right] .
\end{aligned}
$$

Now by using the fact that $A(0,0)=\mathbb{I}$ and the bounds on the derivatives $A$ as in (1.11), we observe that

$$
A \nabla \log G=-\left(\frac{x}{2 t}+\frac{O\left(|x|^{2}+t\right)^{(\beta+1) / 2}}{2 t}\right) .
$$

We note that the first term in the right hand side of (3.12) can be equivalently written as

$$
\begin{aligned}
& \int_{\mathbb{R}_{+}^{n+1}} \frac{\sigma^{1-\alpha}}{\dot{\sigma}}(-f(X, u))\left(\partial_{t} u-<A \nabla \log G, \nabla u>+\frac{1}{2} H u-\frac{\alpha \dot{\sigma}}{2 \sigma} u\right) G d X \\
= & \int_{\mathbb{R}_{+}^{n+1}} \frac{\sigma^{1-\alpha}}{\dot{\sigma}}(-f(X, u))\left(\frac{Z u}{2 t}+\frac{1}{2} H u-\frac{\alpha \dot{\sigma}}{2 \sigma} u\right) G d X,
\end{aligned}
$$


where

$$
Z=2 t\left(-<A \nabla \log G, \nabla>+\partial_{t}\right) .
$$

Now because of (3.13) as well as (1.11), it follows that

$$
\left\{\begin{array}{l}
Z=<x, \nabla>+2 t \partial_{t}+O\left(\left(|x|^{2}+t\right)^{(\beta+1) / 2}\right) \nabla_{x} \\
\operatorname{div}_{X} Z=n+2+O\left(|x|^{\beta}\right)
\end{array}\right.
$$

Now we look at each individual term in the right hand side of (3.14). First we observe that from the following identity

$$
Z F(X, u)=f(X, u) Z u+<Z, \nabla_{X} F>
$$

the first term on the right hand side of (3.14) can be rewritten as

$$
-\int_{\mathbb{R}_{+}^{n+1}} \frac{\sigma^{1-\alpha}}{\dot{\sigma}} f(X, u) \frac{Z u}{2 t} G d X=-\int_{\mathbb{R}_{+}^{n+1}} \frac{\sigma^{1-\alpha}}{\dot{\sigma}} \frac{1}{2 t}\left(Z F(X, u) G-<Z, \nabla_{X} F>G\right) d X .
$$

Now from the bounds in (1.9) we see that the second term in the right hand side of (3.16) can be upper bounded by

$$
C_{2} \int_{\mathbb{R}_{+}^{n+1}} \sigma^{1-\alpha} \frac{1}{2 t}(|x|+|t|) F(X, u) G d X .
$$

Then again by using the first inequality in Lemma 2.4 with $u$ replaced by $\sqrt{F}$, we can assert that this term can by bounded from above in the following way

$$
\begin{aligned}
& \int_{\mathbb{R}_{+}^{n+1}} \sigma^{1-\alpha} \frac{1}{2 t}(|x|+|t|) F(X, u) G d X \leq \int_{\mathbb{R}_{+}^{n+1}} \sigma^{1-\alpha} F(X, u) G d X \\
& +C\left[e^{N \alpha} \gamma^{\alpha+N} \int_{\mathbb{R}_{+}^{n+1}} t F(X, u) d X+\delta^{\beta} \int_{\mathbb{R}_{+}^{n+1}} \sigma^{1-\alpha} \frac{\theta(\gamma t)}{t} F(X, u) G d X\right] .
\end{aligned}
$$

In (3.17), we also used the fact that since $u(\cdot, t)$ is supported in $B_{2}$, therefore $|x| \lesssim|x|^{\beta}$ in the support of $u$.

Now by applying integration by parts to the first integral in the right hand side of (3.16) we obtain

$$
\begin{aligned}
& -\int_{\mathbb{R}_{+}^{n+1}} \frac{\sigma^{1-\alpha}}{\dot{\sigma}} \frac{1}{2 t} Z F(X, u) G d X \\
& =\int_{\mathbb{R}_{+}^{n+1}}\left[\frac{\sigma^{1-\alpha}}{\dot{\sigma}} \frac{1}{2 t} F(X, u)\left(\left(\operatorname{div}_{X} Z\right) G+Z G\right)+F(X, u) G\left(t \partial_{t}\right)\left[\frac{\sigma^{1-\alpha}}{t \dot{\sigma}}\right]\right] d X .
\end{aligned}
$$

At this point, we note that since $G$ is parabolic homogeneous of degree $-n$, therefore we have that

$$
<x, \nabla G>+2 t \partial_{t} G=-n G \text {. }
$$

Then by using this fact, it follows from the expression of $Z$ as in (3.15) that the following holds,

$$
Z G=-\left(n+\frac{O\left(\left(|x|^{2}+t\right)^{1+\beta / 2}\right)}{t}\right) G .
$$

We also have that

$$
\left(t \partial_{t}\right)\left[\frac{\sigma^{1-\alpha}}{t \dot{\sigma}}\right]=(1-\alpha) \sigma^{-\alpha}-\frac{\sigma^{1-\alpha}}{t \dot{\sigma}}-\frac{\sigma^{1-\alpha} \ddot{\sigma}}{(\dot{\sigma})^{2}}
$$


Now note that since $\frac{d}{d t} \log \left(\frac{\sigma}{t \dot{\sigma}}\right)=\frac{\dot{\sigma}}{\sigma}-\frac{1}{t}-\frac{\ddot{\sigma}}{\dot{\sigma}}=\frac{\theta(\gamma t)}{t}$, therefore

$$
\frac{\ddot{\sigma}}{\dot{\sigma}} \lesssim \frac{1}{t}
$$

This implies that

$$
\left(t \partial_{t}\right)\left[\frac{\sigma^{1-\alpha}}{t \dot{\sigma}}\right]=-\alpha \sigma^{-\alpha}+O(1) \sigma^{-\alpha} .
$$

Therefore by using (3.15), (3.19) and (3.22) in (3.18) it follows

$$
\begin{aligned}
& -\int_{\mathbb{R}_{+}^{n+1}} \frac{\sigma^{1-\alpha}}{\dot{\sigma}} \frac{1}{2 t} Z F(X, u) G d X \\
& =\int_{\mathbb{R}_{+}^{n+1}}\left[\frac{\sigma^{1-\alpha}}{\dot{\sigma}}\left(\frac{1}{t}+\frac{O\left(\left(|x|^{2}+t\right)^{1+\beta / 2}\right)}{t}+\frac{O\left(|x|^{\beta}\right)}{t}\right) G F(X, u)+(-\alpha+O(1)) \sigma^{-\alpha} F(X, u) G\right] d X .
\end{aligned}
$$

Now the first term on the right hand side of (3.23) is again estimated by using the inequalities in Lemma 2.4 ( with $\sqrt{F}$ instead of $u$ ) as follows

$$
\begin{aligned}
& \int_{\mathbb{R}_{+}^{n+1}} \frac{\sigma^{1-\alpha}}{\dot{\sigma}}\left(\frac{1}{t}+\frac{O\left(\left(|x|^{2}+t\right)^{1+\beta / 2}\right)}{t}+\frac{O\left(|x|^{\beta}\right)}{t}\right) G F(X, u) d X \\
& \leq C\left[e^{N \alpha} \gamma^{\alpha+N} \int_{\mathbb{R}_{+}^{n+1}} F(X, u) d X+\delta^{\beta} \int_{\mathbb{R}_{+}^{n+1}} \sigma^{1-\alpha} \frac{\theta(\gamma t)}{t} F(X, u) G d X\right. \\
& \left.+\int_{\mathbb{R}_{+}^{n+1}} \sigma^{-\alpha} F(X, u) G d X\right] .
\end{aligned}
$$

Over here we note that in (3.24) above, we used the fact that in the support of $u(\cdot, t)$ which is contained in $B_{2}$, we have

$$
\frac{O\left(\left(|x|^{2}+t\right)^{1+\beta / 2}\right.}{t} \lesssim \frac{O\left(|x|^{\beta}\right)}{t}+O(1) .
$$

We also used the bounds for $\sigma, \dot{\sigma}$ as in Lemma 2.3. Now again by using the bounds for $\sigma, \dot{\sigma}$ as in Lemma 2.3, the bounds for $H$ as in (3.1) and the fact that

$$
0 \leq u f(X, u) \leq q F(X, u)
$$

which is contained in the structural assumptions as in (1.9) ( In fact this is precisely the place where we use the specific structure of the sublinearity), we obtain the following estimate for the last two terms in the right hand side of (3.14),

$$
\begin{aligned}
& \left|\int_{\mathbb{R}_{+}^{n+1}} \frac{\sigma^{1-\alpha}}{\dot{\sigma}}(-f(X, u))\left(\frac{1}{2} H u-\frac{\alpha \dot{\sigma}}{2 \sigma} u\right) G d X\right| \\
& \leq C \int_{\mathbb{R}_{+}^{n+1}} \sigma^{1-\alpha} \frac{\left(|x|^{2}+t\right)^{1+\beta / 2}}{t^{2}} F(X, u) G d X+\frac{\alpha q}{2} \int_{\mathbb{R}_{+}^{n+1}} \sigma^{-\alpha} F(X, u) G d X .
\end{aligned}
$$


Now again by using the inequalities in Lemma 2.4, the first term in the right hand side of (3.25) can be estimated in the following way

$$
\begin{aligned}
& \int_{\mathbb{R}_{+}^{n+1}} \sigma^{1-\alpha} \frac{\left(|x|^{2}+t\right)^{1+\beta / 2}}{t^{2}} F(X, u) G d X \\
& \leq N e^{N \alpha} \gamma^{\alpha+N} \int_{\mathbb{R}_{+}^{n+1}} F(X, u) d X+N \delta^{\beta} \alpha \int_{\mathbb{R}_{+}^{n+1}} \sigma^{1-\alpha} \frac{\theta(\gamma t)}{t} F(X, u) G d X .
\end{aligned}
$$

At this point, by using the estimates (3.17), (3.23), (3.24), (3.25) and (3.26) in (3.12) we obtain

$$
\begin{aligned}
& \int_{\mathbb{R}_{+}^{n+1}} \sigma^{1-\alpha} \frac{\theta(\gamma t)}{t}|\nabla u|^{2} G d X \\
& \leq\left(-\alpha+\frac{q}{2} \alpha+O(1)\right) \int_{\mathbb{R}_{+}^{n+1}} \sigma^{-\alpha} F(X, u) G d X \\
& +C\left[\int_{\mathbb{R}_{+}^{n+1}} \sigma^{1-\alpha}|g|^{2} G d X+\left(\alpha \delta^{\beta}+1\right) \int_{\mathbb{R}_{+}^{n+1}} \sigma^{-\alpha} \frac{\theta(\gamma t)}{t} u^{2} G+\sigma^{-\alpha} F(X, u) G d X\right. \\
& \left.+\delta^{\beta} \int_{\mathbb{R}_{+}^{n+1}} \sigma^{1-\alpha} \frac{\theta(\gamma t)}{t}|\nabla u|^{2} G d X+e^{N \alpha} \gamma^{\alpha+N} \int_{\mathbb{R}_{+}^{n+1}}\left(u^{2}+t|\nabla u|^{2}+F(X, u)\right) d X\right] .
\end{aligned}
$$

Over here we note that in order to get to (3.27) above, we also used the fact that $\frac{\theta(\gamma t)}{t} \lesssim c \sigma^{-1}$. Now the inequality (3.5) can be equivalently written as

$$
\begin{aligned}
& \frac{\alpha}{C_{1}} \int_{\mathbb{R}_{+}^{n+1}} \sigma^{-\alpha} \frac{\theta(\gamma t)}{t} u^{2} G d X \\
& \leq\left[\int_{\mathbb{R}_{+}^{n+1}} \sigma^{1-\alpha} \frac{\theta(\gamma t)}{t}|\nabla u|^{2} G d X+\int_{\mathbb{R}_{+}^{n+1}} \sigma^{1-\alpha}|g|^{2} G d X\right. \\
& \left.e^{N \alpha} \gamma^{\alpha+N} \int_{\mathbb{R}_{+}^{n+1}} u^{2} d X\right] .
\end{aligned}
$$

Now since $q<2$, we can now choose $\alpha$ sufficiently large such that

$$
\left(-\alpha+\frac{q}{2} \alpha+O(1)\right) \leq \frac{1}{4}(q-2) \alpha .
$$

Moreover, we also choose $\delta$ small enough such that

$$
C \delta^{\beta}<\frac{1}{16}, C\left(\alpha \delta^{\beta}+1\right) \leq \min \left(\frac{\alpha}{4 C_{1}}, \frac{1}{8}(2-q) \alpha\right)
$$

where $C$ and $C_{1}$ are the constants as in (3.17) and (3.28) respectively. Now by adding the inequalities $(3.17)+\frac{1}{2}(3.28)$, the desired estimate in Theorem 1.2 follows by also taking into account (3.29) and (3.30).

Before proceeding further, we make the following remark.

Remark 3.1. It remains to be seen whether the Carleman estimate (1.14) holds if we instead assume that $A$ satisfies

$$
|A(x, t)-A(y, s)| \leq C\left(|x-y|^{2}+|t-s|\right)^{1 / 2}
$$


as in Theorem 1 i) in $[\mathrm{EF}]$ ( which constitutes an alternate set of conditions under which the strong unique continuation result in $[\mathrm{EF}]$ is valid) or more generally if $A$ can be allowed to have $1 / 3$ Hölder regularity in time as in $[\mathrm{KT}]$. However the proof of the Carleman estimate in $[\mathrm{EF}]$ for principal part A with regularity assumptions as in (3.31) crucially relies on weighted Calderon Zygmund estimate of the following type

$$
\frac{1}{\delta^{2}} \int \sigma^{2-\alpha}\left|\nabla^{2} u\right|^{2} G d X \lesssim \int \sigma^{1-\alpha}|\operatorname{div}(A(x, 0) \nabla u)|^{2} G d X+e^{N \alpha} \gamma^{\alpha+N} \int\left(u^{2}+t|\nabla u|^{2}\right) G d X
$$

( see for instance (3.9) in $[\mathrm{EF}])$. It remains to be seen whether in our sublinear situation, one can get similar estimates with $|\operatorname{div}(A(x, 0) \nabla u)|^{2}$ replaced by $(\operatorname{div}(A(x, 0) \nabla u)+f(X, u))^{2}$. This appears to be a challenging interesting issue to which we would like to come back in a future study.

Proof of Theorem 1.1. We now proceed with the proof of our main unique continuation result Theorem 1.1.

Proof. The proof is divided into two steps.

Step 1: We first assume that $u$ vanishes to infinite order in space and time at $(0,0)$, i.e. for every $k \in \mathbb{N}$, there exists $C_{k}$ such that

$$
|u(x, t)| \leq C_{k}\left(|x|^{2}+t\right)^{k / 2} .
$$

Also by taking a smaller neighborhood if necessary and then by iteratively spreading the zero set, without loss of generality we may assume that $|u| \leq 1$. Now note that from our regularity assumption on $A$ as in (1.11), it follows from the Calderon-Zygmund estimates as in [Li] that given any $p<\infty$,

$$
\nabla^{2} u, u_{t} \in L^{p}\left(B_{r} \times[0,2)\right)
$$

for all $r<2$. Moreover, we also have from the Schauder theory that $\nabla u$ is in $H_{l o c}^{\beta}\left(B_{2} \times[0,2)\right)$. Now for a given $\varepsilon>0$, let $u_{\varepsilon}=u \phi_{\epsilon}(t) \psi(x)$, where $\psi \in C_{0}^{\infty}\left(\mathbb{R}^{n}\right)$ satisfies $\psi=1$ for $|x| \leq 1, \psi=0$ for $|x| \geq 3 / 2$ and $\phi_{\varepsilon} \in C_{0}^{\infty}(\mathbb{R})$ is a smooth cutoff such that

$$
\left\{\begin{array}{l}
\phi_{\varepsilon} \equiv 1, \text { when } \epsilon \leq t \leq \frac{1}{4 \gamma} \\
\phi_{\varepsilon} \equiv 0, \text { when } t \leq \frac{\epsilon}{2} \text { or } t \geq \frac{1}{2 \gamma} .
\end{array}\right.
$$

Then we have that

$$
\sum_{i, j}^{n} \partial_{i}\left(a_{i j}(X) \partial_{j} u_{\epsilon}\right)+\partial_{t} u_{\epsilon}+f\left(X, u_{\varepsilon}\right)=\tilde{g}_{\varepsilon}
$$

where

$$
\begin{aligned}
& \tilde{g}_{\epsilon}=f\left(X, u_{\varepsilon}\right)-f(X, u) \phi_{\epsilon}(t) \psi(x)-V u \phi_{\varepsilon}(t) \psi(x) \\
& +2 \phi_{\epsilon}(t) \sum_{i, j}^{n} a_{i j}(X) \partial_{j} u \partial_{i} \psi+u \phi_{\epsilon}(t) \sum_{i, j}^{n} \partial_{i}\left(a_{i j}(X) \partial_{j} \psi\right)+u \phi_{\epsilon}^{\prime}(t) \psi(x) .
\end{aligned}
$$

Now given an integer $k \geq \tilde{C}$ (where $\tilde{C}$ is as in Theorem 1.2) we apply the Carleman estimate in Theorem 1.2 with $\alpha=2 k$ to $u_{\epsilon}$ ( note that the validity of the Carleman estimate (1.14) for 
$u_{\varepsilon}$ can be justified using an approximation with smooth functions and also by using (3.34)). Consequently we have

$$
\begin{aligned}
& \alpha \int_{\mathbb{R}_{+}^{n+1}} \sigma^{-\alpha} \frac{\theta(\gamma t)}{t}\left|u_{\epsilon}\right|^{2} G d X+\int_{\mathbb{R}_{+}^{n+1}} \sigma^{1-\alpha} \frac{\theta(\gamma t)}{t}\left|\nabla u_{\epsilon}\right|^{2} G d X \\
& +O(\alpha) \int_{\mathbb{R}_{+}^{n+1}} \sigma^{-\alpha} F\left(X, u_{\varepsilon}\right) G d X \\
& \leq N_{0} \int_{\mathbb{R}_{+}^{n+1}} \sigma^{1-\alpha}\left|\tilde{g}_{\epsilon}\right|^{2} G d X+e^{N_{0} \alpha} \gamma^{\alpha+N_{0}} \int_{\mathbb{R}_{+}^{n+1}}\left(u_{\epsilon}^{2}+t\left|\nabla u_{\epsilon}\right|^{2}+F\left(X, u_{\varepsilon}\right)\right) d X .
\end{aligned}
$$

Now using $u f(X, u) \leq q F(X, u)$ we note that the following Caccioppoli type energy estimate hold

$$
\int_{B_{r} \times\left(a, a+r^{2}\right)}|\nabla u|^{2} d X \leq \frac{C}{r^{2}} \int_{B_{2 r} \times\left(a, a+2 r^{2}\right)}\left(u^{2}+F(X, u)\right) d X .
$$

Now since

$$
F(X, u) \leq c|u|^{p}
$$

for some $p \in(1,2)$, it follows from (3.33), the gradient estimate above and parabolic regularity estimates as in $[\mathrm{Li}]$ that $\nabla u$ also vanishes to infinite order in space and time in the sense of (3.33).

Now by splitting the integrals over $\mathbb{R}^{n} \times(0, \infty)$ into dyadic time-like regions of the type $\mathbb{R}^{n} \times\left\{1 / 2^{k} \leq t \leq 1 / 2^{k-1}\right\}$ and by using vanishing to infinite order property of $u$ and $\nabla u$, we can assert that for any $\alpha>0$,

$$
\int_{B_{3 / 2} \times(0,1)} \sigma^{-\alpha}\left(u^{2}+|\nabla u|^{2}\right) G<\infty .
$$

Moreover using the following bound

$$
\left|\phi_{\varepsilon}^{\prime}(t)\right| \leq \frac{C}{\varepsilon} \text {, when } \frac{\varepsilon}{2}<t<\varepsilon
$$

and (3.33), we note that as $\varepsilon \rightarrow 0$,

$$
\int_{\frac{\varepsilon}{2}<t<\varepsilon} u^{2} \psi^{2} \phi_{\varepsilon}^{\prime}(t)^{2} d X \rightarrow 0 .
$$

Therefore we can let $\varepsilon \rightarrow 0$ in (3.36) and consequently we obtain for $u_{0}=u \phi_{0} \psi$ where $\phi_{0}$ is the pointwise limit of $\phi_{\varepsilon}$ that the following inequality holds, 


$$
\begin{aligned}
& \alpha \int_{\mathbb{R}_{+}^{n+1}} \sigma^{-\alpha} \frac{\theta(\gamma t)}{t}\left|u_{0}\right|^{2} G d X+\int_{\mathbb{R}_{+}^{n+1}} \sigma^{1-\alpha} \frac{\theta(\gamma t)}{t}\left|\nabla u_{0}\right|^{2} G d X+O(\alpha) \int_{\mathbb{R}_{+}^{n+1}} \sigma^{-\alpha} F\left(X, u_{0}\right) G d X \\
& \leq N_{0} \int_{\mathbb{R}_{+}^{n+1}} \sigma^{1-\alpha}\left|\tilde{g}_{0}\right|^{2} G d X+e^{N_{0} \alpha} \gamma^{\alpha+N_{0}} \int_{\mathbb{R}_{+}^{n+1}}\left(u_{0}^{2}+t\left|\nabla u_{0}\right|^{2}+\left|u_{0}\right|^{q}\right) d X \\
& \leq N_{0} \int_{\mathbb{R}_{+}^{n+1}} \sigma^{1-\alpha}\left[\left(f(X, u) \phi_{0} \psi-f\left(X, u_{0}\right)\right)^{2}+|u|^{2} \phi_{0}^{2} \psi^{2}\right] G d X \\
& +2 N_{0} \int_{B_{2} \backslash B_{1} \times(0,1 / 2 \gamma)} \sigma^{1-\alpha}\left|\phi_{0}(t) \nabla u\right|^{2} G d X+N_{0} \int_{B_{2} \backslash B_{1} \times(0,1 / 2 \gamma)} \sigma^{1-\alpha}\left|\phi_{0}(t) u\right|^{2} G d X \\
& +N_{0} \int_{\mathbb{R}_{+}^{n+1}} \sigma^{1-\alpha}\left|u \phi_{0}^{\prime}(t) \psi\right|^{2} G d X+e^{N_{0} \alpha} \gamma^{\alpha+N_{0}} \int_{\mathbb{R}_{+}^{n+1}}\left(u_{0}^{2}+t\left|\nabla u_{0}\right|^{2}+\left|u_{0}\right|^{p}\right) d X
\end{aligned}
$$

where $N_{0}$ additionally depends on the $L^{\infty}$ norm of $V$.

Now we estimate each individual term in the right hand side of the above expression. We first note that from the expression of $\theta$ as in Lemma 2.4 it follows that $\gamma \lesssim \frac{\theta(\gamma t)}{t}$. Consequently using $\gamma=\frac{\alpha}{\delta^{2}}$, we have

$$
\delta^{-2} \lesssim \frac{\theta(\gamma t)}{t}
$$

when $0<2 \gamma t<1$. Therefore we have that the following terms on the right hand side of (3.38)

$2 N_{0} \int_{B_{2} \backslash B_{1} \times(0,1 / 2 \gamma)} \sigma^{1-\alpha}\left|\phi_{0}(t) \nabla u\right|^{2} G d X+N_{0} \int_{B_{2} \backslash B_{1} \times(0,1 / 2 \gamma)} \sigma^{1-\alpha}\left|\phi_{0}(t) u\right|^{2} G d X+N_{0} \int_{\mathbb{R}_{+}^{n+1}} \sigma^{1-\alpha} u^{2} \phi_{0}^{2} \psi^{2} G d X$

can be estimated in the following way

$$
\begin{aligned}
& 2 N_{0} \int_{B_{2} \backslash B_{1} \times(0,1 / 2 \gamma)} \sigma^{1-\alpha}\left|\phi_{0}(t) \nabla u\right|^{2} G d X+N_{0} \int_{B_{2} \backslash B_{1} \times(0,1 / 2 \gamma)} \sigma^{1-\alpha}\left|\phi_{0}(t) u\right|^{2} G d X \\
& +N_{0} \int_{\mathbb{R}_{+}^{n+1}} \sigma^{1-\alpha} u^{2} \phi_{0}^{2} \psi^{2} G d X \\
& \leq C \delta^{2}\left(\int_{B_{2} \times(0,1 / 2 \gamma)} \sigma^{1-\alpha} \frac{\theta(\gamma t)}{t}|\nabla u|^{2} G d X+\int_{B_{2} \times(0,1 / 2 \gamma)} \sigma^{1-\alpha} \frac{\theta(\gamma t)}{t}|u|^{2} G d X\right)
\end{aligned}
$$

where $C$ is independent of $\delta$. Now by choosing $\delta$ sufficiently small, we note that these terms can then be absorbed in the left hand side of (3.38). We consequently fix such a $\delta$. We then consider the following term in the right hand side of (3.38)

$$
\int_{\mathbb{R}_{+}^{n+1}} \sigma^{1-\alpha}\left(f(X, u) \phi_{0} \psi-f\left(X, u_{0}\right)\right)^{2} G d X
$$


Note that this term is non-zero in $B_{2} \backslash B_{1} \times(0,1 / 2 \gamma) \cup B_{2} \times(1 / 4 \gamma, 1 / 2 \gamma)$. Therefore this term can be estimated from above in the following way using $e^{-N} t \leq \sigma \leq t$,

$$
\begin{aligned}
& \int_{\mathbb{R}_{+}^{n+1}} \sigma^{1-\alpha}\left(f(X, u) \phi_{0} \psi-f\left(X, u_{0}\right)\right)^{2} G d X \\
& \leq C e^{2 N k}\left(\int_{B_{3 / 2} \backslash B_{1} \times(0,1 / 2 \gamma)} t^{-2 k+1}|u|^{2 p-2} G d X+\int_{B_{3 / 2} \times(1 / 4 \gamma, 1 / 2 \gamma)} t^{-2 k+1}|u|^{2 p-2} G d X\right)
\end{aligned}
$$

where in (3.41) above, we used that $|f(\cdot, s)| \leq \kappa|s|^{p-1}$ for some $p \in(1,2)$. Now since $\alpha=2 k$ and $\gamma=\frac{\alpha}{\delta^{2}}$, it follows that if $|x| \geq 1$ or $t \geq 1 / 4 \gamma$, there exists $N$ depending also on $\delta$ such that

$$
t^{-2 k} G \leq e^{2 N k} k^{2 k} \text {. }
$$

Also from Stirling's formula, we have

$$
k^{k} \lesssim e^{N k} k !
$$

Therefore for a new $N$, we obtain

$$
\begin{aligned}
& \int_{\mathbb{R}_{+}^{n+1}} \sigma^{1-\alpha}\left(f(X, u) \phi_{0} \psi-f\left(X, u_{0}\right)\right)^{2} G d X \\
& \leq C e^{2 N k}(k !)^{2}\left(\int_{B_{3 / 2} \backslash B_{1} \times(0,1 / 2 \gamma)}|u|^{2 p-2} d X+\int_{B_{3 / 2} \times(1 / 4 \gamma, 1 / 2 \gamma)}|u|^{2 p-2} d X\right) .
\end{aligned}
$$

Likewise the term

$$
N_{0} \int_{B_{2} \backslash B_{1} \times(0,1 / 2 \gamma)} \sigma^{1-\alpha}\left|\phi_{0}(t) u\right|^{2} G d X
$$

can be bounded from above in the following way

$$
N_{0} \int_{B_{2} \backslash B_{1} \times(0,1 / 2 \gamma)} \sigma^{1-\alpha}\left|\phi_{0}(t) u\right|^{2} G d X \leq C e^{2 N k}(k !)^{2} \int_{B_{3 / 2} \backslash B_{1} \times(0,1 / 2 \gamma)} u^{2} d X .
$$

Then we observe that the term

$$
N_{0} \int_{\mathbb{R}_{+}^{n+1}} \sigma^{1-\alpha}\left|u \phi_{0}^{\prime}(t) \psi\right|^{2} G d X
$$

is estimated from above as follows

$$
N_{0} \int_{\mathbb{R}_{+}^{n+1}} \sigma^{1-\alpha}\left|u \phi_{0}^{\prime}(t)\right|^{2} G d X \leq N \int_{B_{2} \times(1 / 4 \gamma, 1 / 2 \gamma)} \sigma^{1-\alpha}|u|^{2} G d X \lesssim e^{2 N k}(k !)^{2} \int_{B_{2} \times(1 / 4 \gamma, 1 / 2 \gamma)} u^{2} d X
$$

where in (3.44) above, we again made use of Stirling formula. Finally the following term in the right hand side of (3.38)

$$
e^{N_{0} \alpha} \gamma^{\alpha+N_{0}} \int_{\mathbb{R}_{+}^{n+1}}\left(u_{0}^{2}+t\left|\nabla u_{0}\right|^{2}+\left|u_{0}\right|^{p}\right) d X
$$

is handled as follows

$$
e^{N_{0} \alpha} \gamma^{\alpha+N_{0}} \int_{\mathbb{R}_{+}^{n+1}}\left(u_{0}^{2}+t\left|\nabla u_{0}\right|^{2}+\left|u_{0}\right|^{p}\right) d X \leq e^{2 N k}(k !)^{2} \int_{B_{3 / 2} \times(0,1 / 2 \gamma)}\left(u^{2}+t|\nabla u|^{2}+|u|^{p}\right) d X .
$$


Now by using the energy estimate as in (3.37), we can assert that the following inequality holds,

$$
e^{N_{0} \alpha} \gamma^{\alpha+N_{0}} \int_{\mathbb{R}_{+}^{n+1}}\left(u_{0}^{2}+t\left|\nabla u_{0}\right|^{2}+\left|u_{0}\right|^{p}\right) d X \leq e^{2 N k}(k !)^{2} \int_{B_{2} \times(0,2)}|u|^{p} d X
$$

for some $N$. Note that in order to get to (3.46), we used the Stirling formula and also the fact that since $u$ is bounded, therefore $u^{2} \lesssim|u|^{p}$. Therefore, by combining (3.38), (3.40), (3.42), (3.43), (3.44) and (3.46) we finally obtain for a new $N$ and $k \geq \tilde{C}$ that the following holds,

$$
\int_{B_{1} \times(0,4 / \gamma)} t^{-2 k} u^{2} G d X \leq\left(\frac{1}{4} N\right)^{2+2 k}(k !)^{2} \int_{B_{2} \times(0,2)}|u|^{2(p-1)} d X .
$$

Note that in (3.47) we also used the boundedness of $u$ and the fact that since $p \in(1,2)$, therefore

$$
2(p-1)=\min (2(p-1), p, 2) .
$$

Now by writing

$$
\int_{B_{1} \times(0,1)} t^{-2 k} u^{2} G d X=\int_{B_{1} \times(0,4 / \gamma)} t^{-2 k} u^{2} G d X+\int_{B_{1} \times(4 / \gamma, 1)} t^{-2 k} u^{2} G d X
$$

and by estimating

$$
\int_{B_{1} \times(4 / \gamma, 1)} t^{-2 k} u^{2} G d X
$$

using

$$
t^{-2 k} G \lesssim e^{2 N k} k^{2 k}, \text { since } t \geq 4 / \gamma
$$

we consequently obtain using Stirling formula and (3.47) that for a new $N$, the following estimate holds for $k \geq \tilde{C}$,

$$
\left(\int_{B_{1} \times(0,1)} t^{-2 k} u^{2} G d X\right)^{1 / 2} \leq\left(\frac{1}{4} N\right)^{1+k}(k !)\|u\|_{L^{\infty}\left(B_{2} \times(0,2)\right)}^{(p-1)} .
$$

Now by multiplying the inequality by $2^{k} / N^{k} k$ ! and summing over $k \geq \tilde{C}$, we obtain

$$
\sum_{k \geq \tilde{C}}\left(\int_{B_{1} \times(0,1)} \frac{2^{k}}{N^{k} t^{k} k !} u^{2} G d X\right)^{1 / 2} \leq N\|u\|_{L^{\infty}\left(B_{2} \times(0,2)\right)}^{p-1} .
$$

Now we note that there exists $K_{0}$ depending on $\tilde{C}$, such that for $a \geq K_{0}$,

$$
\sum_{k \geq \tilde{C}} \frac{a^{k}}{k !} \geq \frac{1}{2} e^{a} .
$$

Consequently we have from (3.49), (3.50) and triangle inequality that the following holds,

$$
\left(\int_{B_{1} \times\left(0, \frac{2}{N K_{0}}\right)} e^{2 / N t} u^{2} G d X\right)^{1 / 2} \lesssim\|u\|_{L^{\infty}\left(B_{2} \times(0,2)\right)}^{p-1} .
$$

Now using

$$
\frac{1}{N t} \geq \frac{|x|^{2}}{8 t}-\frac{|x-y|^{2}}{8 t}
$$


when $|y| \leq 8 / N$ and $|x| \leq 1 / 2$, we obtain from (3.51) and the explicit expression of $G(x, t)$ that

$$
\left(\int_{B_{1} \times(0, s)} t^{-n / 2} u^{2} e^{-\frac{|x-y|^{2}}{4 t}} d X\right)^{1 / 2} \lesssim e^{-1 / N s}\|u\|_{L^{\infty}\left(B_{2} \times(0,2)\right)}^{p-1}
$$

for $s \in\left(0, \frac{1}{N K_{0}}\right)$ and $y$ such that $|y| \leq 8 / N$. Therefore it follows from (3.52) that for all such $s, y$ we have

$$
\left(\int_{B_{\sqrt{s}}(y) \times(s, 2 s)} s^{-n / 2} u^{2} d X\right)^{1 / 2} \lesssim e^{-1 / N s}\|u\|_{L^{\infty}\left(B_{2} \times(0,2)\right)}^{p-1} .
$$

Now note that since $u$ solves (1.8), therefore by treating $f(X, u)$ as a scalar term and by applying the standard Moser subsolution estimate as in [AS] ( see also Theorem 6.29 in [Li]), we obtain

$$
|u(y, s)| \lesssim \frac{1}{s^{n / 2+1}} \int_{s}^{2 s} \int_{B_{\sqrt{s}}(y)}|u| d X+s^{1-n / 2 q_{0}}\left(\int_{s}^{2 s} \int_{B_{\sqrt{s}}(y)} u^{(p-1) q_{0}} d X\right)^{1 / q_{0}}, q_{0}>n / 2+1 .
$$

Here we also used that $f(\cdot, s) \lesssim|s|^{p-1}$ as in (1.10). We additionally choose $q_{0}$ large enough such that $(p-1) q_{0} \geq 2$. Now by Cauchy-Schwartz and (3.53), the first integral in the right hand side of (3.54) is upper bounded by $e^{-1 / N s}\|u\|_{L^{\infty}\left(B_{2} \times(0,2)\right)}^{(p-1) / 2}$ and for the second integral, since $(p-1) q_{0} \geq 2$ and $u$ is bounded, therefore the second integral on the right hand side of (3.54) can be estimated as follows

$$
s^{1-n / 2 q_{0}}\left(\int_{s}^{2 s} \int_{B_{\sqrt{s}}(y)} u^{(p-1) q_{0}} d X\right)^{1 / q_{0}} \leq C\left(\int_{s}^{2 s} \int_{B_{\sqrt{s}}(y)} u^{2} d X\right)^{1 / q_{0}}
$$

where $C$ depends on the $L^{\infty}$ norm of $u$ which again because of (3.53) can be upper bounded by $e^{-1 / N s}\|u\|_{L^{\infty}\left(B_{2} \times(0,2)\right)}^{2(p-1) / q_{0}}$ for a different $N$. Therefore finally we obtain that for some universal $N$ that $u$ satisfies the following estimate

$$
|u(y, s)| \leq C e^{-1 / N s} \text {, when }|y| \leq 8 / N, s<\frac{1}{N K_{0}} .
$$

This implies that $u(\cdot, 0) \equiv 0$ in $B_{8 / N}$ and the estimate (3.56) in particular implies that $u$ vanishes to infinite order in space and time at every $(y, 0)$ for $|y| \leq 8 / N$. At this point, by a standard argument we can spread the zero set and conclude that $u(\cdot, 0) \equiv 0$.

Step 2: We now show that if $u$ vanishes to infinite order at $(0,0)$ in the space variable in the sense of Definition 2.5, then $u$ also vanishes to infinite order in both space and time in the sense of (3.33). For linear parabolic equations, this follows from a result of Alessandrini and Vessella in $[\mathrm{AV}]$. We note that proof in $[\mathrm{AV}]$ uses the local asymptotics of solutions to parabolic equations vanishing to a certain order in space and time as derived in [AV1]. However the proof of such a local asymptotic result in [AV1] relies on certain scaling properties of a linear equation in a crucial way and this is not available in our sublinear situation. Therefore we instead adapt an alternate approach due to Fernandez in $[\mathrm{F}]$.

We proceed as follows. We note that it suffices to show (3.33) for $k$ large enough. Let $k \geq M$ where $M$ is large enough to be decided later. We additionally assume that $M \geq \tilde{C}$ where $\tilde{C}$ is as in Theorem 1.2. Corresponding to this $k$, as before let $\alpha=2 k$ and $\gamma=\frac{\alpha}{\delta^{2}}$ where $\delta$ is small 
enough as required in Step 1 . Now for a fixed $a$ such that $0<a<\frac{1}{4 \gamma}$ and with $\psi, \phi_{0}$ as in Step 1 corresponding to such a $\gamma$, by repeating the arguments in the proof of Theorem 1.2 to $u_{0}=u \psi \phi_{0}$ in the region $\mathbb{R}_{+}^{n+1}$ with $G(x, t+a)$ instead of $G(x, t)$ and $\sigma(t+a)$ instead of $\sigma(a)$, and by keeping track of the additional positive boundary terms which occur when integrating by parts with respect to the time-variable and then by adding up such terms to the right hand side of our previous estimate (3.38), we note that after such a computation, the additional boundary integrals on the right hand side (i.e. at $\mathbb{R}^{n} \times\{0\}$ ) are bounded from above by a multiple of

$$
\alpha \sigma(a)^{-\alpha} \int_{\mathbb{R}^{n} \times\{0\}} u_{0}^{2}(x, 0)\left(1+\frac{|x|^{2}}{a}\right) G(x, a) d x+\sigma(a)^{-\alpha} \int_{\mathbb{R}^{n} \times\{0\}} F\left((x, 0), u_{0}(x, 0)\right) G(x, a) d x .
$$

We note that the first integral above is as in $[\mathrm{F}]$ ( see Section 3 in $[\mathrm{F}]$ ) whereas the second integral is the one that is incurred due to an integration by parts of an expression involving the sublinear term as in (3.18). Then by using

$$
F(\cdot, s) \leq C s^{p}, \text { for some } p \in(1,2)
$$

we see that the expression in (3.57) is upper bounded by

$$
C \alpha \sigma(a)^{-\alpha} \int_{\mathbb{R}^{n} \times\{0\}}\left|u_{0}\right|^{p}(x, 0)\left(1+\frac{|x|^{2}}{a}\right) G(x, a) d x .
$$

Now by repeating the arguments as in Step 1 upto (3.48) we obtain the following estimate for some universal $N$ ( Over here, note that the inequality (3.39) still holds since $a \leq 1 / 4 \gamma$ )

$$
\int_{B_{1} \times(0,1)}(t+a)^{-2 k} u^{2} G(x, t+a) d X \leq N^{2 k}(k !)^{2}\|u\|_{L^{\infty}\left(B_{2} \times(0,2)\right)}^{2(p-1)}+N^{2 k} a^{-2 k} \int_{B_{2} \times\{0\}}|u|^{p}(x, 0) G(x, a) d x .
$$

Now since $a \leq \frac{1}{4 \gamma}$ and $\gamma \sim k$, therefore, we have that $a \leq \frac{1}{C k}$. Now note that (3.58) in particular implies the following estimate

$$
\int_{B_{\sqrt{a}} \times(0, a)} u^{2} d X \leq N^{2 k}(k !)^{2} a^{2 k}|| u \|_{L^{\infty}\left(B_{2} \times(0,2)\right)}^{2(p-1)}+N^{2 k} \int_{B_{2} \times\{0\}}|u|^{p}(x, 0) G(x, a) d x .
$$

Now given some $a \in\left(0, \frac{1}{C k}\right)$, using the fact that $u$ vanishes to infinite order in space, we can ensure that

$$
N^{2 k} \int_{B_{2} \times\{0\}}|u|^{p}(x, 0) G(x, a) d x \leq M_{k} a^{k} .
$$

Now again by repeating the arguments as in (3.54)-(3.55) which uses the Moser's subsolution estimate and also by using (3.60) we can assert that there exists universal constants $N_{1}, N_{2}>0$ such that for all $k \geq N_{1}$, we have

$$
\|u\|_{L^{\infty}\left(B_{\sqrt{a} / 2} \times[0, a / 2)\right)} \leq M_{k} a^{k / N_{2}}, \text { for some } M_{k} \text { and where } a \leq \frac{1}{C k} .
$$

Now it can be seen by a standard real analysis argument that (3.61) implies (3.33) and consequently by Step 1, we can again conclude that $u(\cdot, 0) \equiv 0$. This completes the proof of the Theorem. 


\section{REFERENCES}

[Al] F. J. Almgren, Jr., Dirichlet's problem for multiple valued functions and the regularity of mass minimizing integral currents. Minimal submanifolds and geodesics, (Proc. Japan-United States Sem., Tokyo, 1977), pp. 1-6, North-Holland, Amsterdam-New York, 1979.

[ACS] I. Athanasopoulos, L. A. Caffarelli \& S. Salsa, The structure of the free boundary for lower dimensional obstacle problems, Amer. J. Math. 130 (2008), no. 2, 485-498.

[AKS] Aronszajn, N. , Krzywicki, A \& Szarski, J. A unique continuation theorem for exterior differential forms on Riemannian manifolds,Ark. Mat. 41962 417-453 (1962).

[AS] D. Aronson \& J. Serrin, Local behavior of solutions of quasilinear parabolic equations, Arch. Rational Mech. Anal. 251967 81-122.

[AV] G. Alessandrini \& S. Vessella, Remark on the strong unique continuation property for parabolic operators, Proc. Amer. Math. Soc. 132 (2004), no. 2, 499-501.

[AV1] _ Local behaviour of solutions to parabolic equations, Comm. Partial Differential Equations 13 (1988), no. $9,1041-1058$.

[BGR] A. Banerjee, N. Garofalo \& R. Manna, New Carleman estimates for Baouendi-Grushin operators with applications to quantitative uniqueness and strong unique continuation, preprint

[Car] T. Carleman, Sur un probleme d'unicite pur les systemes d'equations aux derivees partielles a deux variables independantes, Ark. Mat., Astr. Fys. 26 (1939). no. 17, 9 pp

[Ch] X. Chen, A strong unique continuation theorem for parabolic equations, Math. Ann., 311 (1998), 603-630.

[CS] S. Chanillo \& E. Sawyer, Unique continuation for $\Delta+\nu$ and the C. Fefferman-Phong class, Trans. Amer. Math. Soc. 318 (1990), no. 1, 275-300.

[CSS] L. A. Caffarelli, S. Salsa \& L. Silvestre, Regularity estimates for the solution and the free boundary of the obstacle problem for the fractional Laplacian, Invent. Math. 171 (2008), no. 2, 425-461.

[E] L. Escauriaza, Carleman inequalities and the heat operator, Duke Math. J. 104 (2000), no. 1, 113-127.

[EF] L. Escauriaza \& F. Fernandez, Unique continuation for parabolic operators. (English summary), Ark. Mat. 41 (2003), no. 1, 35-60.

[EFV] L. Escauriaza, F. Fernandez \& S. Vessella, Doubling properties of caloric functions, Appl. Anal. 85 (2006), no. 1-3, 205-223.

[ESS] L. Escauriaza, G. Seregin \& V. Sverak, Backward uniqueness for parabolic equations, Arch. Ration. Mech. Anal. 169 (2003), no. 2, 147-157.

[EV] L. Escauriaza \& L. Vega, Carleman inequalities and the heat operator. II. (English summary) Indiana Univ. Math. J. 50 (2001), no. 3, 1149-1169.

[F] F. Fernandez, Unique continuation for parabolic operators II, Comm. Partial Differential Equations 28 (2003), no. 9-10, 1597-1604.

[GG] N. Garofalo \& M.S.V Garcia, New monotonicity formulas and the optimal regularity in the Signorini problem with variable coefficients, Adv. Math. 262 (2014), 682-750.

[GL1] N. Garofalo \& F. Lin, Monotonicity properties of variational integrals, $A_{p}$ weights and unique continuation, Indiana Univ. Math. J. 35 (1986), 245-268.

[GL2] Unique continuation for elliptic operators: a geometric-variational approach, Comm. Pure Appl. Math. 40 (1987), 347-366.

[J] F. Jones, A fundamental solution for the heat equation which is supported in a strip, J. Math. Anal. Appl. $60(1977)$

[JK] D. Jerison \& C. Kenig, Unique continuation and absence of positive eigenvalues for Schrodinger operators, Ann. of Math. (2) 121 (1985), no. 3, 463-494.

[K] G. Karadzhov, Riesz summability of multiple Hermite series in $L^{p}$ spaces, C.R. Acad. Bulgare Sci., 47 (1994), 5-8.

[KRS] C. Kenig, A. Ruiz \& C. Sogge, Uniform Sobolev inequalities and unique continuation for second order constant coefficient differential operators, Duke Math. J. 55 (1987), no. 2, 329-347.

[KT0] H. Koch \& D. Tataru, Carleman estimates and unique continuation for second-order elliptic equations with nonsmooth coefficients, Comm. Pure Appl. Math. 54 (2001), no. 3, 339-360.

[KT] H. Koch \& D. Tataru, Carleman estimates and unique continuation for second order parabolic equations with nonsmooth coefficients (English summary), Comm. Partial Differential Equations 34 (2009), no. 4-6, 305-366.

[L] F. Lin, A uniqueness theorem for parabolic equations, Comm. Pure Appl. Math., 43 (1990), 127-136.

[Li] G. Lieberman, Second order parabolic differential equations, World Scientific Publishing Co., Inc., River Edge, NJ, 1996. xii+439 pp. ISBN: 981-02-2883-X 
[Mi] S. Mizohata, Unicite du prolongement des solutions pour quelques operateurs differentiels paraboliques, Mem. Coll. Sci. Univ. Kyoto. Ser. A. Math., 31 219-239.

[Pl] A. Plis, On non-uniqueness in Cauchy problem for an elliptic second order differential equation, Bull. Acad. Polon. Sci. Ser. Sci. Math. Astronom. Phys. 111963 95?100.

[Po] C. C. Poon, Unique continuation for parabolic equations, Comm. Partial Differential Equations 21 (1996), no. 3-4, 521-539.

[PW] E. Parini \& T. Weth, Existence, unique continuation and symmetry of least energy nodal solutions to sublinear Neumann problems, Math. Z, 280 (2015), 707-732.

[Ru] A. Ruland, Unique Continuation for Sublinear Elliptic Equations Based on Carleman Estimates, arXiv: 1801.05563

[So] C. Sogge, A unique continuation theorem for second order parabolic differential operators, Ark. Mat. 28 (1990), 159-182.

[ST] N. Soave \& S. Terracini, The nodal set of solutions to some elliptic problems: sublinear equations, and unstable two-phase membrane problem, arXiv:1802.02089

[SW] N. Soave \& T. Weth, The unique continuation property of sublinear equations, arXiv:1707.07463

[T] S. Thangavelu, Summability of Hermite expansions. I, II, Trans. Amer. Math. Soc. 314 (1989), no. 1, 119-142, 143-170.

[Vaz] J. Vazquez, The porous medium equation: mathematical theory, Oxford University press, 2007.

[WZ] J. Wu \& L. Zhang, Backward uniqueness of parabolic equations with variable coefficients in a half space, Commun. Contemp. Math. 18 (2016), no. 1, 38 pp.

[We] G. Weiss, A homogeneity improvement approach to the obstacle problem, Invent. Math. 138 (1999), no. 1, 23-50.

[Y] H. Yamabe, A unique continuation theorem of a diffusion equation, Ann. of Math.(2) 69 (1959), $462-466$.

Tata Institute of Fundamental Research, Centre For Applicable Mathematics, Bangalore560065, INDIA

E-mail address, Agnid Banerjee: agnidban@gmail.com

Tata Institute of Fundamental Research, Centre For Applicable Mathematics, Bangalore560065, INDIA

E-mail address, Ramesh Manna: ramesh@tifrbng.res.in 